\title{
Neutron transmission probability through a revolving slit for a continuous source and a divergent neutron beam
}

J. Peters*, Hahn-Meitner-Institut Berlin, Glienicker Str. 100, D - 14109 Berlin

\section{Abstract:}

Here an analytical calculation for the transmission probability of neutrons travelling through a revolving slit is being presented. For the first time, two effects have been taken into account in the same approach, that is on the one hand the fact that the neutron beam might be divergent and, on the other hand, that neutrons from a continuous source can arrive at different times at the chopper. Furthermore, the neutron distribution at a given distance behind the chopper has been calculated and these theoretical results have been compared with simulated data obtained with the VITESS simulation program. The theoretical and the simulated curves are in good agreement.

PACS: 61.12.Ld, 07.05.Fb

Keywords: Fermi chopper, neutron diffraction, transmission probability

*Tel. number (030) 8062 3068, email: peters@hmi.de (J. Peters) 


\section{Introduction}

Some decades ago, in the 1940ies, E. Fermi, J. Marshall and L. Marshall [1] proposed a new kind of velocity selector or rotating shutter to chop the continuous neutron beam into short pulses, thus obtaining a well defined wavelength-time correlation. The first rotating shutter has been further extended, improved and described by T. Brill and H.V. Lichtenberger [2]. This kind of chopper, composed of a sandwich package of neutron transmitting and neutron absorbing foils such as aluminium and cadmium placed into a fast revolving cylinder, is now often referred to as "Fermi chopper". These early works contain mostly mechanical and technical descriptions of the chopper and experimental results.

In the 1950ies, the shape of a neutron burst travelling through a single revolving slit has been derived analytically for different cases: P.A. Egelstaff [3] has provided the formula for the transmission probability of a divergent beam of neutrons arriving all at equal time at the chopper centre. He introduced his formalism from the perspective of the rotor's frame of reference, where the neutrons move on a parabolic curve. Marseguerra and Pauli [4] presented a theory for a curved parabolic Fermi chopper with a parallel beam of neutrons passing through the centre of the chopper at different times (this applies, for instance, to a continuous source). They used the frame of reference of the neutrons. Later, Tolk \& Brugger and Royston [5] studied two consecutive revolving slits placed at a given distance from each other, whereas Beckurts \& Wirtz [6] summarised concisely some analytical results and applications.

Whenever neutron instruments meet the requirement of high resolution in time or in energy (for instance IN4 or IN6 at ILL/France, Pharos at LANL/USA, HET at ISIS/UK), the Fermi chopper can be very useful. Furthermore, the technique is now so powerful due to magnetic bearings as to allow very high repetition rates per minute (up to $36.000 \mathrm{RPM}$ ) and a very 
precise phasing [7]. When a Fermi chopper is placed in such an instrument, it is used to produce time bursts of well defined time and wavelength intervals. But in practice, the neutron beam is always divergent and the neutrons passing through the slit are distributed in time. To describe the two effects simultaneously, a unification of both formalisms is necessary and this can only be done within one frame of reference.

In this paper, such an analytical calculation of the transmission probability will be presented and confronted with data obtained from the Monte Carlo simulation program VITESS written by the Hahn-Meitner-Institut (HMI) simulation group [8]. It can be shown that they are in good agreement. The Fermi chopper characteristics will be such as they will be implemented at the new Extreme Environment Diffractometer (EXED), for which this study has been undertaken. The analytical calculations compared to simulations of the complete instrument allow not only for a benchmarking of the simulation program, but enable us to evaluate the resolution of the instrument [9] and to optimise the total chopper system, which is composed of the Fermi chopper and six disc choppers. Three of the disc choppers are frame overlap choppers and avoid contaminations by higher orders and therefore have to be placed at well defined positions. The only way to calculate them is by using the exact knowledge of the length of the chopper pulses.

\section{Transmission probability through a Fermi chopper}

\section{a) Straight Fermi chopper, non-divergent neutron beam, all neutrons pass through the central plane of the chopper $(x=0)$ at equal time $t$}

Let us first briefly recall the analytical approach [4] to calculate the transmission probability through a straight Fermi chopper. In the following we will assume a chopper whose slits all 
have the same length [see Fig. 1] and we will suppose that all neutrons are travelling through the central plane of the chopper $(x=0)$ at the time $t=0$. The chopper is rotating with the angular velocity $\omega$.

\section{Figure 1}

The slit length $2 R$ is assumed to be much longer than the slit height $h$, so that we can use the same approximations as in [4]. The neutron transmission probability amplitude corresponds to the neutrons travelling through the green surface in Fig. 2.

\section{Figure 2}

The transmission probability per time unit for a neutron of velocity $v$ to be transmitted through the revolving system is thus given by the ratio (the neutron wavelength $\lambda$ being related to $v$ through $\lambda[\AA ̊ ́ f]=0.395610^{4} / v[\mathrm{~m} / \mathrm{s}]$

$$
P(v)=\frac{h-\frac{\omega R^{2}}{v}}{h}=1-\frac{\omega R^{2}}{h v}=1-\frac{v_{m}}{v}=1-\frac{\lambda}{\lambda_{m}},
$$

where $v_{m}$ stands for the minimum velocity required to pass through the Fermi chopper, for which $P(v)$ vanishes, and $\lambda_{m}$ is the corresponding wavelength. For the maximum transmission probability, one evaluates the corresponding time of flight $\Delta t=h /(R \omega)$. The transmission probability has thus the form of the right half plane of a triangle with the maximum at the origin (see also Fig. 4).

\section{b) Straight or curved parabolic Fermi chopper, non-divergent neutron beam, the neutrons pass through the central plane of the chopper at different times $t$}

Following the derivation in [4], the formalism can be generalised to a parabolic chopper, from where the straight chopper can be gained as a limiting case. The curvature of the slit will now 
follow the trajectory of a neutron with a given velocity $v_{0}$ and will be passing through the centre of the slit at $t=0$. However, since the slit has a finite height $h$, some neutrons with a velocity $v \neq v_{0}$ and passing through $x=0$ at $t \neq 0$ will also be transmitted. Thus the transmission probability can be calculated as a function of $k$ which depends on the deviation of $v$ from the ideal value $v_{0}$ :

$$
\frac{1}{k}=\frac{1}{v}-\frac{1}{v_{0}}
$$

The case of a straight Fermi chopper can thus be obtained in the limit $v_{0} \rightarrow \infty$.

Contrary to the case symmetric around $x=0$, the transmission probability now explicitly depends on the time $t$, at which the neutrons pass through the plane $x=0$ (see Fig. 3 for example).

\section{Figure 3}

The equations to describe the transmission probability can be found in Table 1 of [4].

Equation (1) can be obtained in the limiting case where $P(v)=\lim _{\substack{t \rightarrow 0 \\ v_{0} \rightarrow \infty}} P(k, t)$.

The transmission probability is represented in Fig. 4 for a straight and a curved Fermi chopper; they only differ in the fact that one package has a curvature with a radius of 214.4

mm, which corresponds to an optimum transmitted wavelength of $2.5 \AA$ at 36.000 RPM. The curved Fermi chopper permits an exact definition of the wavelength to be transmitted with highest probability for a given angular velocity. Especially when $R$ increases, the curved package can be used to limit the wavelength band.

\section{Figure 4}




\section{c) Straight or curved parabolic Fermi chopper, divergent neutron beam, the neutrons pass through the centre of the chopper at different times $t$}

If the neutron beam is divergent, the neutrons can travel through the chopper following oblique paths. These paths, however, are longer, so that neutrons with a velocity below a critical value cannot pass through the chopper any more. In consequence, the transmission probability is decreasing. The base of the triangle representing the transmission probability $P(k, t)$ is proportional to the angle $\alpha_{e s c}$ through which a slit can turn while neutrons are being transmitted (see Fig. 5).

\section{Figure 5}

A maximum escape angle becomes possible if the gradient of the curves $y^{*}$ is less than or equal to the gradient of the neutron path at $x= \pm R$ and is $\alpha_{\text {esc }}=h / R$ [3]. In general, this condition is dependent on velocity and time and requires a careful distinction between cases.

- $\mathbf{T}=\mathbf{0}$

Having suggested first that $t=0$, the gradient of the neutron path becomes $h / 2 R$ and the gradient of the curves $\mathrm{y}^{*}$ becomes $2 \omega R / k$ at $x=+R$. Thus, one finds the maximum escape angle for velocities from $+\infty$ down to $v_{c}$ (see [3]):

$$
v_{c}=\frac{4 \omega R^{2}}{h} .
$$

If the gradient of $y^{*}$ is greater than $h / 2 R$, the escape angle is reduced. One calculates the gradient of $y^{*}$ to be $2 \omega r / k$ at every point $r$ of the curve. If the neutron hits the curve $y_{u}{ }^{*}$ at the point $r$ (becomes tangent) then it leaves the chopper slit at $y=\frac{4 \omega R r}{k}$ with respect to $y_{l}{ }^{*}$ (see

Fig. 6). The equation of the tangent at any point $r$ can thus be written: 


$$
y(x)=\frac{2 \omega r x}{k}-\frac{\omega r^{2}}{k}+\frac{h}{2} .
$$

From eq. (4) one obtains $y_{1}$, where the tangent meets $x=R$,

$$
y_{1} \equiv y(R)=\frac{2 \omega r R}{k}-\frac{\omega r^{2}}{k}+\frac{h}{2}
$$

and the interval $\Delta y$ (see Fig. 6):

\section{Figure 6}

$$
\Delta y=y_{u}^{*}(+R)-y_{1}=\frac{\omega}{k}(R-r)^{2} .
$$

The sum of $\Delta y+\frac{4 \omega R r}{k}=h$, from which one derives an expression for $r$ :

$$
r=\sqrt{\frac{k h}{\omega}}-R
$$

Thus, the escape angle is found to be:

$$
\alpha_{e s c}=\frac{4 \omega R r}{k} \frac{1}{R}=\frac{4 \omega}{k}\left(\sqrt{\frac{k h}{\omega}}-R\right),
$$

and has a maximum value at $k=v_{c}$. The minimum neutron velocity $v_{m}$ to pass through the chopper is obtained for $\alpha_{e s c}=0^{\circ}$ and again results in (see eq. (1)):

$$
v_{m}=\frac{\omega R^{2}}{h}=\frac{v_{c}}{4}
$$

The normalised transmission probability is proportional to the transmission amplitude and to the escape angle and can be expressed in terms of $v_{m}$ :

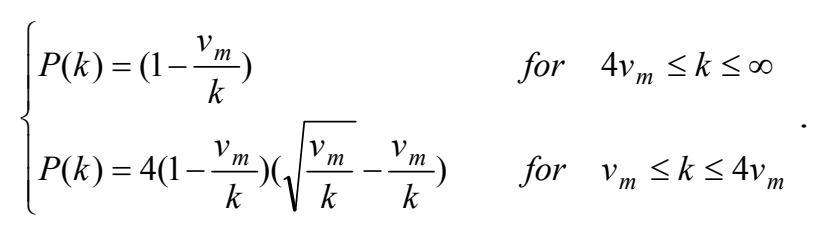


For neutrons which pass through the central plane of the chopper at a time different from zero, we have to distinguish three possible cases. As the gradient of the curves $y_{u, l} *$ is now $2 \omega R / k+\omega t$, the gradient of the neutron path $h / 2 R$ can become smaller than this even for neutron velocities greater than $v_{c}$, for times $t$ greater than a certain limiting value.

(i) Let us first consider a gradient of the neutron path greater than the gradient of $y_{u, l} *$ (see Fig. 7). The point $r_{i}$ can be determined as intersection point of the functions $f(x)$ and $g(x) . F(x)$ and $g(x)$ are both linear functions, which are written for positive times $t$ :

\section{Figure 7}

$$
\left\{\begin{array}{l}
f(x)=\frac{h}{2 R} x+\frac{\omega R^{2}}{k}+\omega t R \\
g(x)=\left(\omega t-\frac{h}{2 R}\right) x+\frac{\omega R^{2}}{k}
\end{array} .\right.
$$

The point $r_{i}$ is then obtained by setting $f\left(r_{i}\right)=g\left(r_{i}\right)$ :

$$
r_{i}=\frac{\omega t R}{\omega t-h / R}
$$

The escape angle is calculated to be

$$
\alpha_{e s c}^{i}=\frac{h}{R+\left|r_{i}\right|}
$$

It can be easily proved that the expressions of $r_{i}$ and the escape angle $\alpha_{e s c}^{i}$ are still valid for the case of negative times $t$, provided that $t$ is substituted by $|t|$ in eqs. (12) and (13). For $r_{i} \rightarrow$ 0 , one finds again that $\alpha_{e s c}^{\max }=\alpha_{e s c}^{i}\left(r_{i}=0\right)=h / R$ as it should. From the condition $\frac{h}{2 R} \geq \frac{2 \omega R}{k}+\omega|t|$, it follows that $|t| \leq \frac{h}{2 R \omega}-\frac{2 R}{k}$ for this case. 
(ii) If now $\frac{h}{2 R}<\frac{2 \omega R}{k}+\omega t$ for positive times $t$, the escape angle $\alpha_{e s c}$ is again reduced. First we have to determine the point $r^{i i}{ }_{1}$ (see Fig. 8), where the neutron path becomes tangent to the curve $y_{u}{ }^{*}$, to find the gradient of the function $h(x)$. Then we have to determine $r^{i i}$, to calculate the escape angle $\alpha^{i i}{ }_{\text {esc }}$.

\section{Figure 8}

To determine $r^{i i}$, one calculates the equation of the tangent $y(x)$ at $x=+R$ to get:

$$
y_{1} \equiv y(R)=\frac{2 \omega R r_{1}^{i i}}{k}-\frac{\omega\left(r_{1}^{i i}\right)^{2}}{k}+\omega t R+\frac{h}{2} .
$$

With the help of this expression and similar to eq. (6), one finds furthermore

$$
\Delta y=\frac{\omega}{k}\left(R-r_{1}^{i i}\right)^{2}
$$

The sum of $\Delta y+\frac{4 \omega R r_{1}^{i i}}{k}+2 \omega R t=h$, from where one gets:

$$
r_{1}^{i i}=\sqrt{\frac{(h-2 \omega R t) k}{\omega}}-R .
$$

In the limiting case $t \rightarrow 0$ one obtains correctly eq. (7).

The linear functions $h(x)$ and $k(x)$ can now be derived:

$$
\left\{\begin{array}{l}
h(x)=\left(\frac{2 \omega r_{1}^{i i}}{k}+\omega t\right) x+\frac{2 \omega r_{1}^{i i} R}{k}+\frac{\omega R^{2}}{k}+2 \omega t R-\frac{h}{2} \\
k(x)=\left(\omega t-\frac{h}{2 R}\right) x+\frac{\omega R^{2}}{k}
\end{array}\right.
$$

and permit to determine the intersection point $r^{i i}{ }_{2}$ :

$$
r_{2}^{i i}=\frac{\frac{h}{2}-\frac{2 \omega r_{1}^{i i} R}{k}-2 \omega R t}{\frac{2 \omega r_{1}^{i i}}{k}+\frac{h}{2 R}}
$$


Similar to eq. (8), one can now calculate the escape angle:

$$
\alpha_{e s c}^{i i}=\frac{\frac{4 \omega r_{1}^{i i} R}{k}+2 \omega R t}{\left|r_{2}^{i i}\right|+R} .
$$

It can be easily proved that the expressions of $r^{i i}$ and the escape angle $\alpha_{\text {esc }}^{i i}$ are still valid for the case of negative times $t$, provided that $t$ is substituted by $|t|$ in eqs. (16), (18) and (19). From the condition $\frac{h}{2 R}<\frac{2 \omega R}{k}+\omega t$, it follows that $|t|>\frac{h}{2 R \omega}-\frac{2 R}{k}$ for this case and thus the limiting case $t \rightarrow 0$ can never be reached.

Case (i) and case (ii) can both be realised for neutron velocities $k>v_{c}$, which one applies depending on the time $t$, when the neutrons pass through the central plane of the chopper. If $k$ $<v_{c}$, the neutron paths cannot become tangent to $y_{u}{ }^{*}$ at $x=+R$ anymore. Here we find a new situation:

(iii) For $k<v_{c}$, we find two linear functions $l(x)$ and $m(x)$ corresponding to the limiting neutron paths (see Fig. 9), which become tangential to $y_{u}{ }^{*}$ at points $r^{i i i}{ }_{1}$ and $r^{i i i}{ }_{2}$ absolutely smaller than $R$.

\section{Figure 9}

First one has to determine the points $r^{i i i}{ }_{1}$ and $r^{i i i}{ }_{2}$ as described above, then the functions $l(x)$ and $m(x)$ can be obtained in a similar way as before to find finally the intersection point $r^{i i i}{ }_{3}$. One gets: $r_{1}^{i i i}=r_{1}^{i i}$ (see eq. (16)), $r^{i i i}{ }_{2}=-r$ (see eq. (7)),

$$
r_{3}^{i i i}=\frac{-R\left(r_{1}^{i i i}+r_{2}^{i i i}+|t| k\right)}{\left(r_{1}^{i i i}-r_{2}^{i i i}\right)}
$$

and 


$$
\alpha_{e s c}^{i i i}=\frac{\frac{4 \omega r_{1}^{i i i} R}{k}+2 \omega R|t|}{\left|r_{3}^{i i i}\right|+R} .
$$

We can check the limiting case $t \rightarrow 0: r^{i i i}{ }_{1} \rightarrow \sqrt{\frac{k h}{\omega}}-R, r_{2}^{i i i} \rightarrow-r^{i i i}{ }_{1}$ and $r^{i i i}{ }_{3} \rightarrow 0$ as it should. In consequence, one finds $\alpha_{e s c}^{i i i} \rightarrow \frac{4 \omega}{k}\left(\sqrt{\frac{k h}{\omega}}-R\right)$ for $t \rightarrow 0$.

There is another point to mention here, and it applies to all cases (i) - (iii): the triangles, through which one determines the escape angles $\alpha_{e s c}^{i}-\alpha_{e s c}^{i i i}$, are not oblique. Consequently, one should calculate the escape angles through the cosine law. However, since it still holds that hypotenuse and adjacent side are much smaller than the opposite side, one can use the following in very good approximation: $\tan \alpha_{\text {esc }} \approx \alpha_{\text {esc }} \approx \frac{\text { opposite side }}{\text { adjacent side }}$.

Furthermore, we used in section (c) above $k, 1 / k=1 / v-1 / v_{0}$, to define the deviation from the optimal velocity $v_{0}$, instead of the true neutron velocity $v$ to obtain general formulas for a curved or a straight Fermi chopper. We have already mentioned, that the velocity $v$ may range from zero to infinity, whereas $k$ can be positive or negative. All equations derived here are also valid for negative $k$, provided that $k$ is replaced by $|k|$. We will summarise the results in Table 1:

Table 1 


\section{Calculated and simulated neutron burst shapes produced by a realistic Fermi chopper}

The analytical results will now be applied to the Fermi chopper, which will be realised at the new Extreme Environment Diffractometer (EXED) of the HMI. When a Fermi chopper is used at a steady state source of neutrons, like for instance the reactor BERII at the HMI, the neutrons are arriving continuously at the chopper, if no other velocity selector is placed before it. At a given moment the Fermi chopper is oriented in a certain direction with respect to the neutron beam. The angle between these two directions will be called the phase angle $\phi_{0}$ (see Fig. 10) of the chopper. This is one of the parameters of the Fermi chopper module in VITESS.

Figure 10

Both the transmission probability $P(k, t)$ and the escape angle $\alpha_{e s c}(k, t)$ are independent functions of the neutron velocity $k$ and the time $t$, when the neutrons travel through the centre of the chopper. However, for a divergent beam, new transmission possibilities exist as the chopper rotates independently from $t$, which means that the chopper may be by chance oriented in a favourable direction with respect to the divergent beam (see Fig. 11). Although the transmission probability is reduced here for a non-divergent neutron beam, the escape angle may achieve a maximum value, i.e. $h / R$, for a divergent beam.

\section{Figure 11}

To take this effect into account, one has to convolute both functions, the convolution integral having as limits the minimum and maximum time the chopper needs to rotate from the minimum to the maximum divergence angle: 


$$
F(v, t)=\int_{\tau_{\min }=\frac{\phi_{\min }}{\omega}}^{\tau_{\max }=\frac{\phi_{\max }}{\omega}} P(k, t-\tau) \alpha_{e s c}(k, \tau) d \tau
$$

If one wants now to compare theory and simulated data, one has to be aware that in the experiment one has only access to the distribution shown by the neutrons after a flight distance $d$ (see Fig. 10). This function will be called $A(d, T)$ as in [4] giving the number of neutrons reaching the plane $x=d$ at the time $T$ :

$$
A(d, T)=\int_{0}^{\infty} F\left(v, t+\frac{d}{v}\right) d v .
$$

A series of cases will be presented now corresponding to the projected Fermi chopper having two slit packages at the instrument EXED of the HMI, showing that the analytical approach derived above is in agreement with simulated calculations in various situations. The real Fermi chopper will be situated at $21.8 \mathrm{~m}$ behind the moderators, but for the calculations the Fermi chopper will be placed immediately behind the source. The instrument will be built at a multispectral beam extraction system and thus will have a view on both thermal and cold moderator. In consequence wavelengths between 0.7 and 20 Á can be achieved here.

At EXED, the neutron pulses will be produced alternatively by a high speed double disc chopper, which permits to have a much higher flux at the cost of resolution, or by the Fermi chopper, which reduces the flux by a factor of about 10, but allows for a much better resolution. The Fermi chopper will be equipped with two slit packages rotating on the same axis. It will be installed on a lift system which enables to place one of the two slit packages into the neutron beam or to replace the whole Fermi chopper by a guide piece and to operate only the double disc chopper. One slit package is straight, the other one has a curvature radius of $214.4 \mathrm{~m}$, which corresponds to an optimum wavelength of $2.5 \AA$ at $36.000 \mathrm{RPM}$. The simulation of the instrument, including the HMI source, and both moderators shows that the 
neutron flux has two local maxima in this guide: one at $1.4 \AA \hat{\text { and the other one at } 3.8 \AA \AA}$. The choice of the curvature radius corresponds to an average wavelength value, for which the flux is very high. The slit packages have a cuboids form with the dimensions of 6 x $10 \times 1 \mathrm{~cm}^{3}$ (width $\mathrm{x}$ height $\mathrm{x}$ depth) for the straight package and of $6 \times 10 \times 2.5 \mathrm{~cm}^{3}$ for the curved package. These dimensions will also be used for the calculations. The distance behind the chopper considered here is $d=7.5 \mathrm{~cm}$. The simulations have been done with the instrument simulation program VITESS [8] written by an HMI group. For each curve 5 million trajectories (= statistical events) were started and some ten thousand of them typically passed through the chopper.

In the following, all transmission probability curves are normalised as to have the maximum at unity. Fig. 12 shows the case of the straight Fermi chopper rotating at 36.000 RPM, neutrons arriving at any time with a divergence of $0.1^{\circ}$ with a fixed wavelength of $\lambda_{0}=1.2 \AA$. For such a small divergence of the neutron beam and a fixed wavelength, one finds the typical triangular form of the burst shape (for instance, see the right-hand side of Fig. 4). The full width half maximum (FWHM) was evaluated to $14 \mu$ s.

Figure 12

Fig. 13 differs from the previous one by corresponding to a wavelength bandwidth of $0.7 \AA \leq$ $\lambda \leq 1.7 \AA$ and has a FWHM of $19.6 \mu \mathrm{s}$. Already at a distance of $7.5 \mathrm{~cm}$ behind the chopper, the curve is enlarged by the different flight times corresponding to the various wavelengths.

\section{Figure 13}

Fig. 14 and 15 show the same Fermi chopper and the same wavelength bandwidth, but the divergence of the incoming neutron beam corresponds now to $1^{\circ}$ and $3^{\circ}$, respectively. As the instrument EXED will be equipped with a guide having coatings up to $m=3$ and the 
multispectral beam extraction system allowing for wavelengths up to $20 \AA \AA$, divergences up to $3.8^{\circ}$ are realistic.

\section{Figure 14}

The higher the divergence, the more the smoothing effect of the convolution (see eq. (23)) becomes visible. The curves have FWHM of $20.6 \mu$ s and $28 \mu$ s and are also described by the theory introduced here.

\section{Figure 15}

Finally, two results corresponding to the curved slit package at 36.000 RPM with $h / R=0.04$ are shown. Only for this case, the difference between $k$ and $v$ (see eq. (2)) becomes important. To avoid unequal integration intervals, one has to integrate over $k$ instead of $v$ in eq. (24) taking into account the corresponding transformation factor.

Fig. 16 is similar to the case shown in Fig. 12 with a fixed wavelength. Due to the depth of the chopper of $2 R=2.5 \mathrm{~cm}$, the FWHM is reduced now to $5.8 \mu \mathrm{s}$. The curved slit package will be used to produce shortest neutron bursts, which make highest resolution possible at the instrument [9]. Here again one finds the typical triangular form.

\section{Figure 16}

Fig. 17 finally offers the neutron pulse for a wavelength bandwidth typically used on EXED of $1.7 \AA \leq \leq \lambda \leq 2.5 \AA$ and a divergence of $1^{\circ}$, which enlarges the curve at a FWHM of $14.4 \mu \mathrm{s}$.

Figure 17 


\section{Conclusion}

In this work it has been shown how to calculate analytically the neutron burst shape produced by a rotating Fermi chopper under the condition of a divergent incoming neutron beam and a continuous flux in time. The calculations are compared to Monte Carlo simulation results for various situations and are in good agreement for both a straight slit package and a curved slit package. The comparison with experimental data will be presented in a separate publication [10] for the data obtained at HET in ISIS. 


\section{References}

[1] E. Fermi, J. Marshall \& L. Marshall, Phys. Rev. 72 (1947) 193 - 196.

[2] T. Brill \& H.V. Lichtenberger, Phys. Rev. 72 (1947) 585 - 590.

[3] P.A. Egelstaff, AERE N/R 1131 (1953) 1 - 38.

[4] M. Marseguerra \& G. Pauli, Nucl. Instr. Meth. 4 (1959) 140 - 150.

[5] N.H. Tolk \& R.M. Brugger, Nucl. Instr. Meth. 8 (1960) 203 - 210, R.J. Royston, Nucl. Instr. Meth. 30 (1964) 184 - 202.

[6] K.H. Beckurts \& K. Wirtz, Neutron Physics, Springer Verlag, 1964.

[7] K.T. Sundset et al., Proc. of the Eighth International Symposium on Magnetic Bearings (ISMB 8), 2002, Mito, Ibaraki-Pref., Japan.

[8] VITESS group: G. Zsigmond, K. Lieutenant, F. Mezei, HMI Berlin, http://www.hmi.de/projects/ess/vitess/

[9] J. Peters, K. Lieutenant, D. Clemens \& F. Mezei, Proc. of the European Powder Diffraction Conference, 2004, submitted for publication.

[10] J. Peters, G. Zsigmond, H. N. Bordallo, J.D.M. Champion \& F. Mezei, to be published. 
Figures and Tables

Figure 1: Schematic design of a Fermi chopper rotor

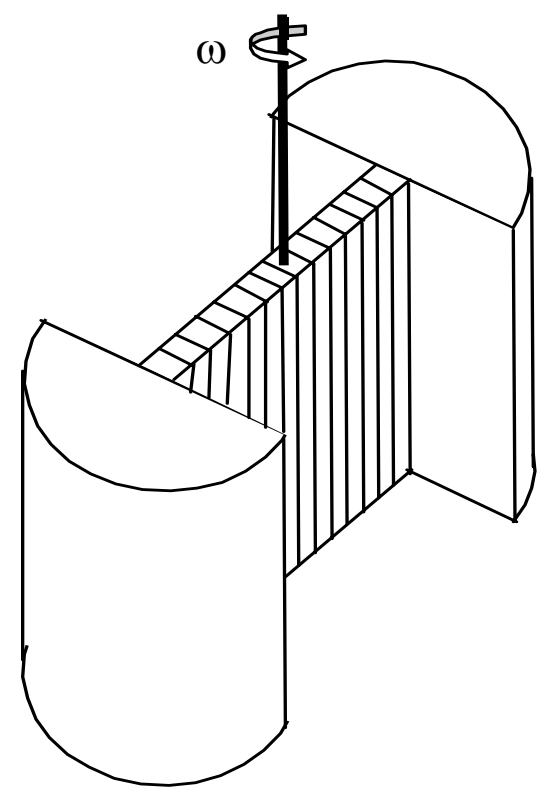

Figure 2: Cross sections of the chopping surfaces with the plane $(\mathrm{x}, \mathrm{y}) \cdot y_{u}^{*}$ and $y_{l}^{*}$ indicate the position of the upper and lower surface of the revolving slit at the transit time of a neutron. A nondivergent neutron beam can pass through the Fermi chopper only in the green dashed sector.

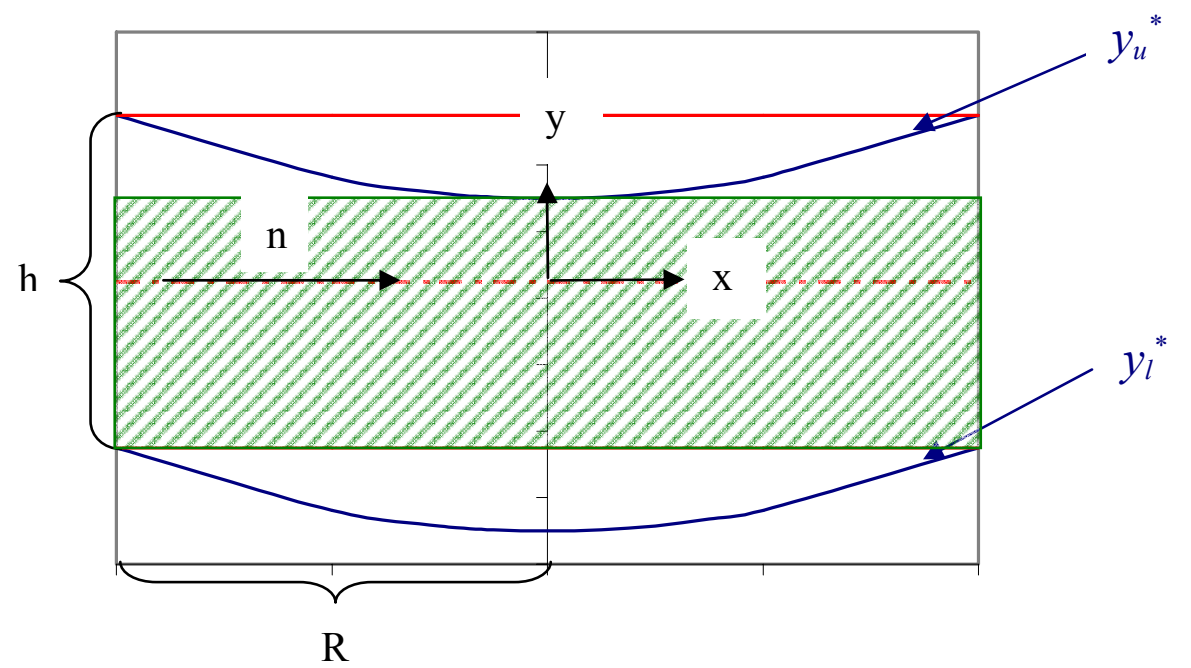


Figure 3: Case of a non-divergent neutron beam and of neutrons which pass through the central plane $x=0$ of the Fermi chopper at a negative time $t$.

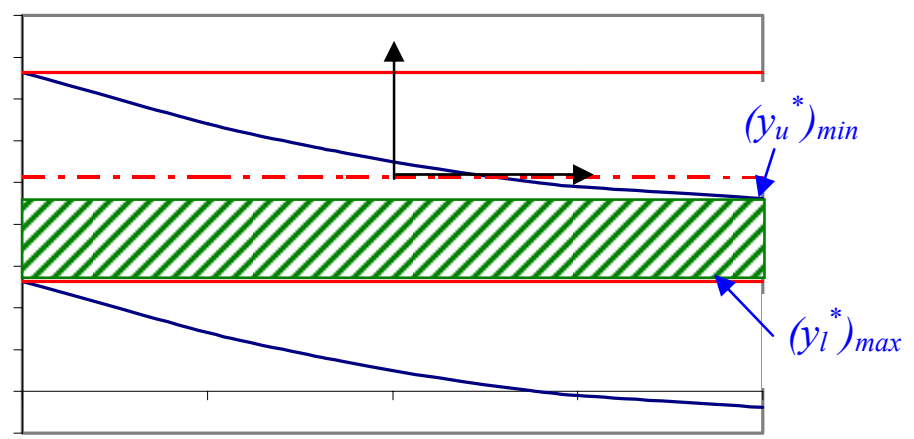

Figure 4: Transmission probabilities as function of the neutron wavelength (velocity) for $t$ fixed (lhs) at zero and as function of the time at a fixed wavelength (rhs). The red line corresponds to a straight package and the blue line to a curved Fermi chopper package. The chopper characteristics are as follows: $R=0.005 \mathrm{~m}, h=0.0005 \mathrm{~m}, f=600 \mathrm{~Hz}$. For both curves, the value of $\lambda_{f i x}=1.2 \AA$ in the rhs figure.
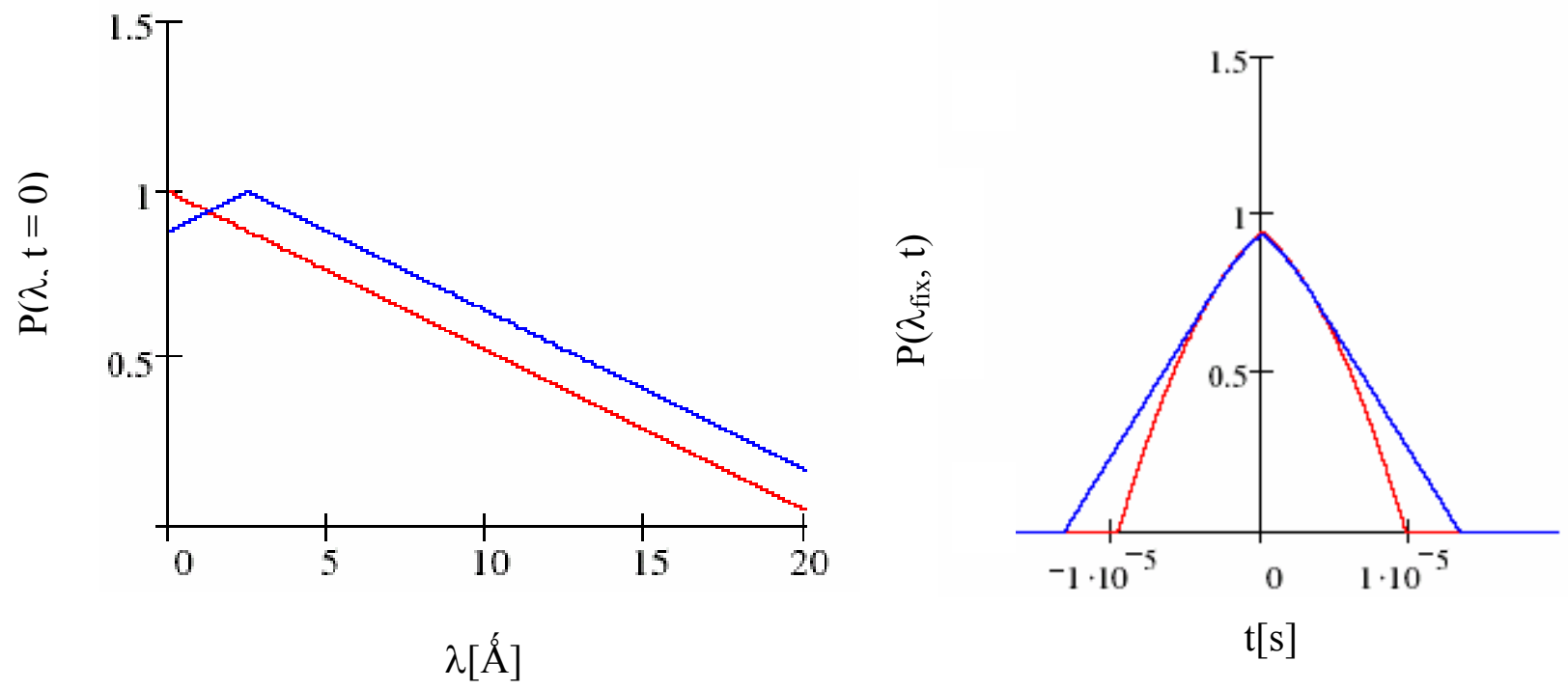
Figure 5: Divergent neutron beam passing through the central plane of the Fermi chopper at $t=0$ with a maximum escape angle.

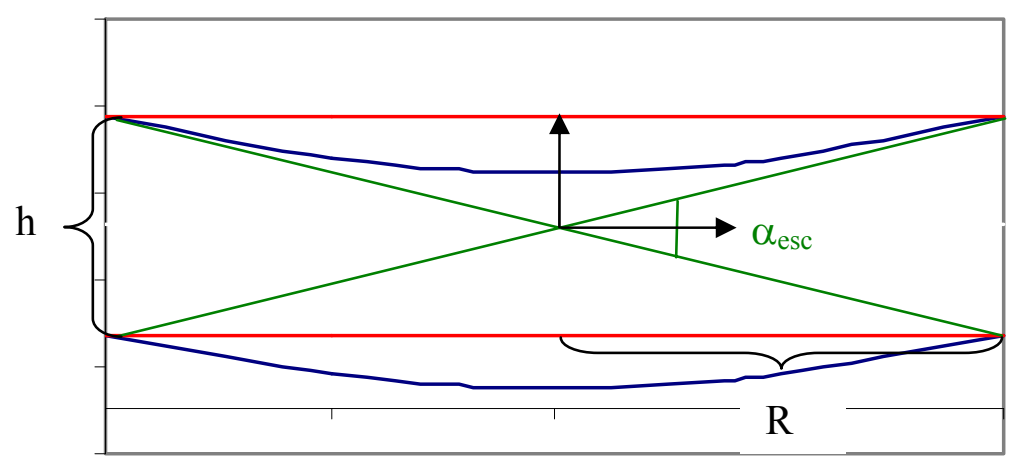

Figure 6: Divergent neutron beam passing through the central plane of the Fermi chopper at $t=0$ with a velocity $k<v_{c}$ and a reduced escape angle.
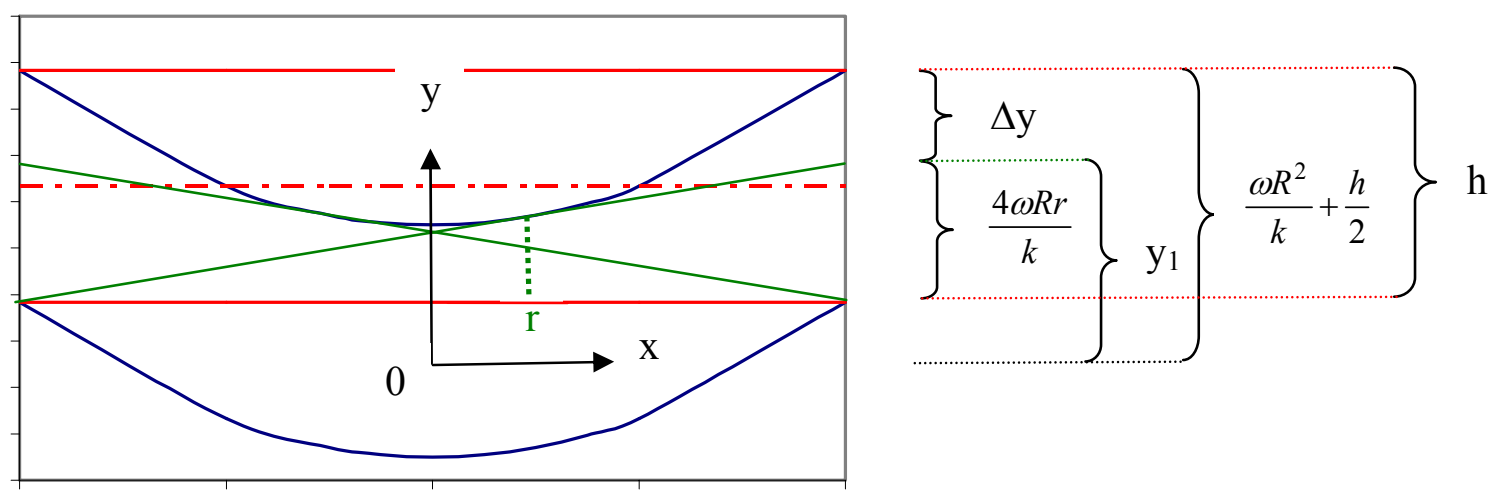

Figure 7: Divergent neutron beam passing through the central plane of the Fermi chopper at $t>0$.

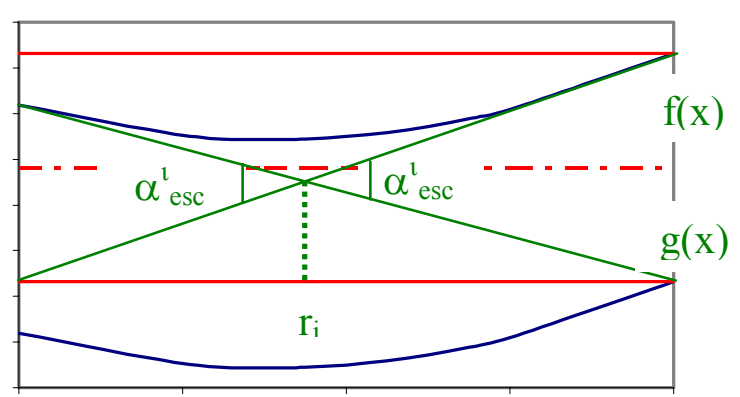


Figure 8: Divergent neutron beam passing through the centre of the Fermi chopper at $t \neq 0$ with a velocity $k>v_{c}$ and a reduced escape angle.

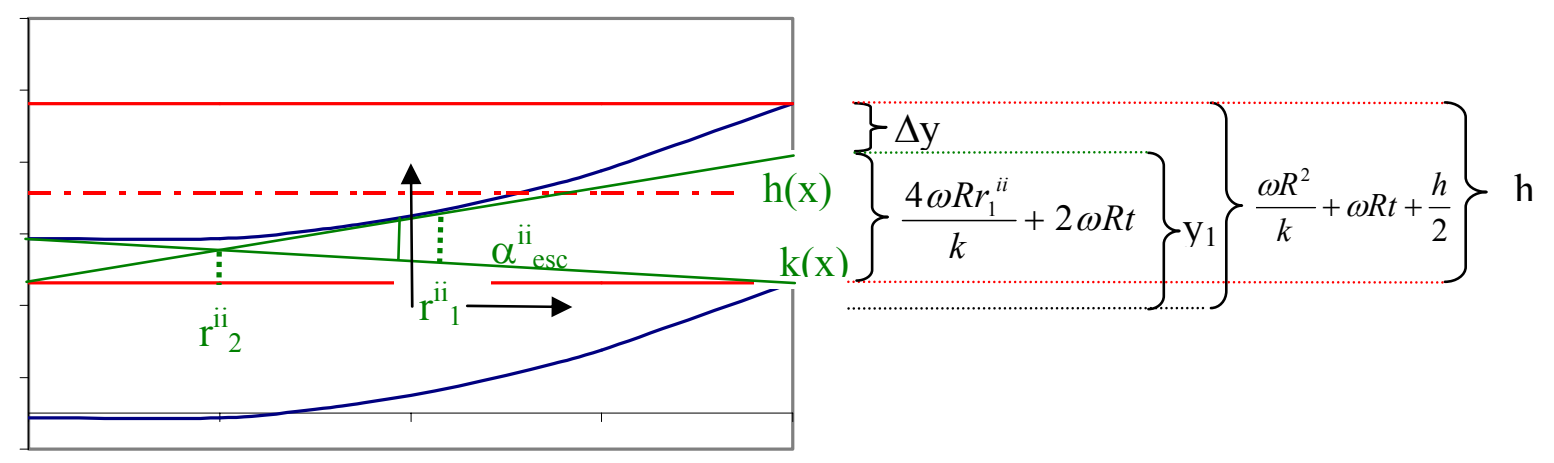

Figure 9: Divergent neutron beam with velocity $k<v_{c}$ passing through the central plane of the Fermi chopper at $t>0$.

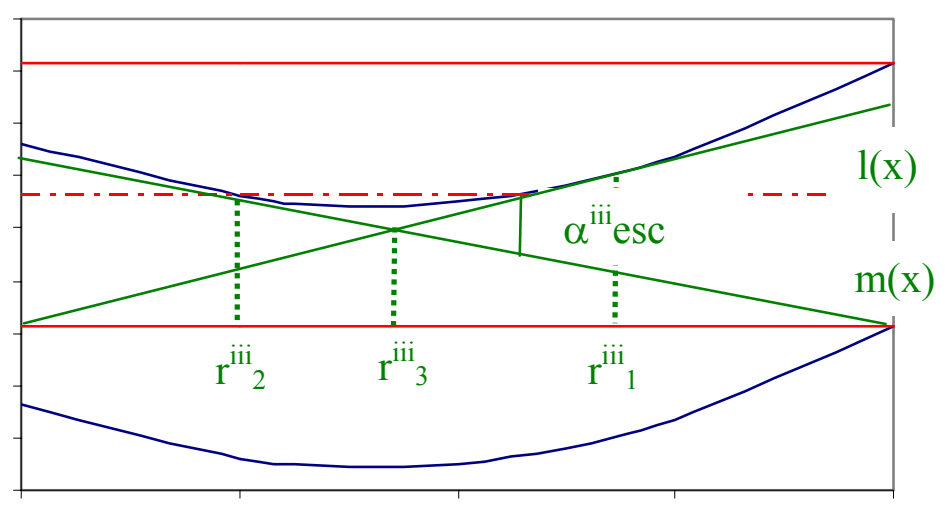


Figure 10: Passage of a neutron beam through the Fermi chopper, which is rotated by the angle $\phi_{0}$ with respect to the beam .

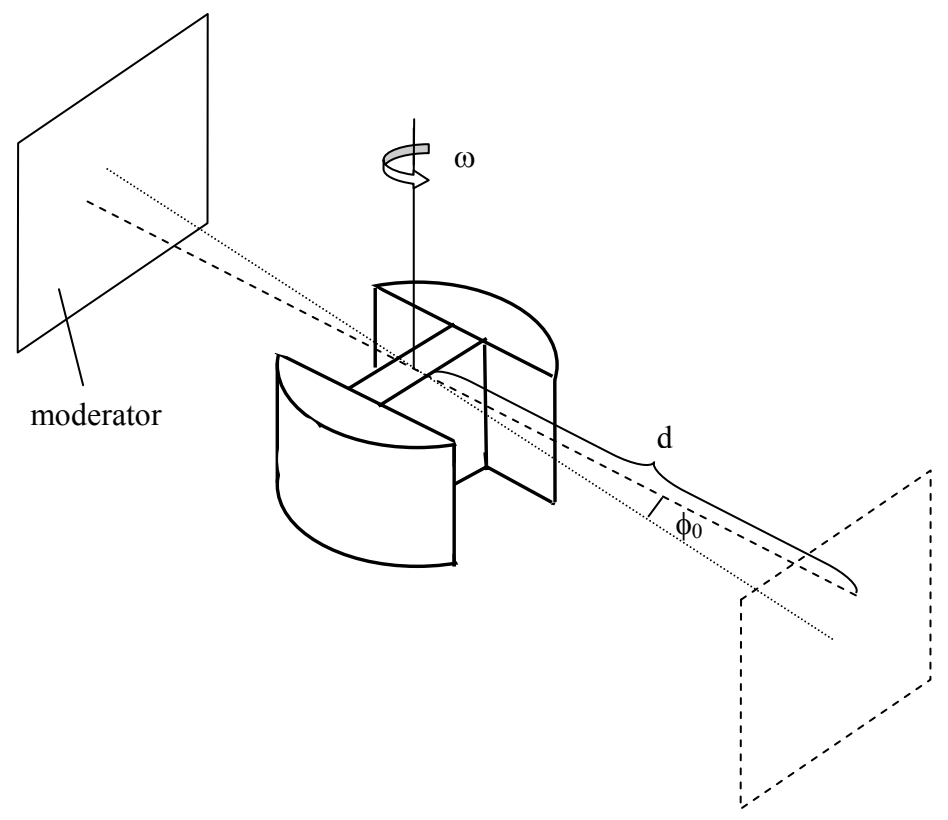

Figure 11: Divergent neutron beam passing through the Fermi chopper. If the chopper is parallel to the beam at $t=0$ for non-divergent straight neutrons, it cannot be simultaneously parallel to the divergent neutrons at $t=0$.

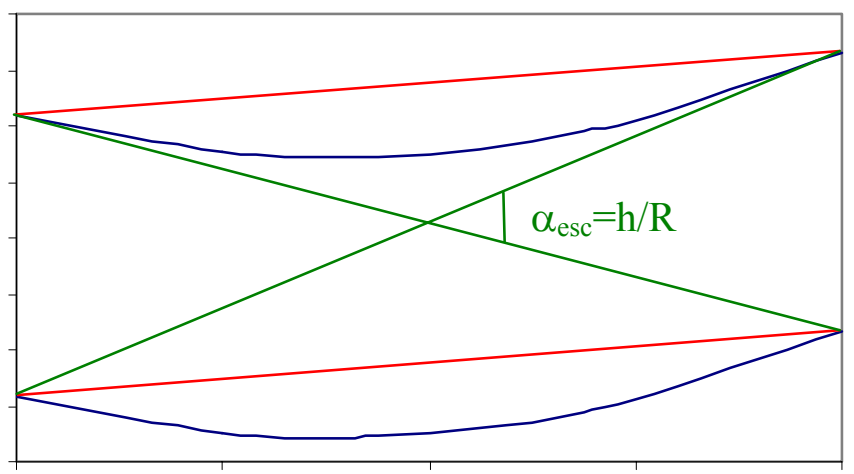


Figure 12: Calculated (straight line) and simulated (scatter points) time distribution curve at a distance $d$ after the passage through a straight Fermi chopper. Here $\lambda_{0}=1.2 \AA$, the divergence $=0.1^{\circ}$.

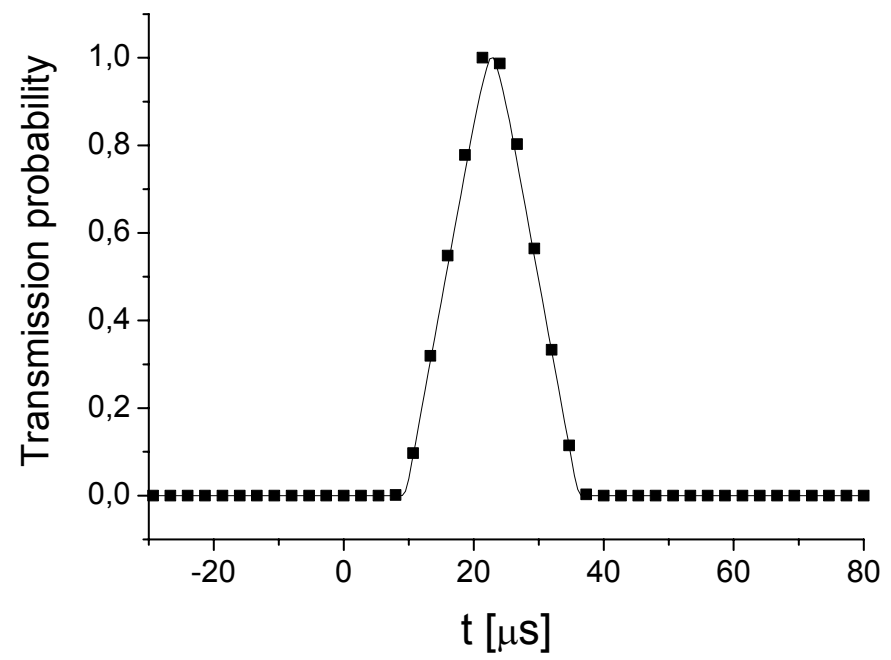

Figure 13: Straight Fermi chopper rotating at 36.000 RPM. Here $0.7 \AA \leq \lambda \leq 1.7 \AA$, the divergence $=$ $0.1^{\circ}$

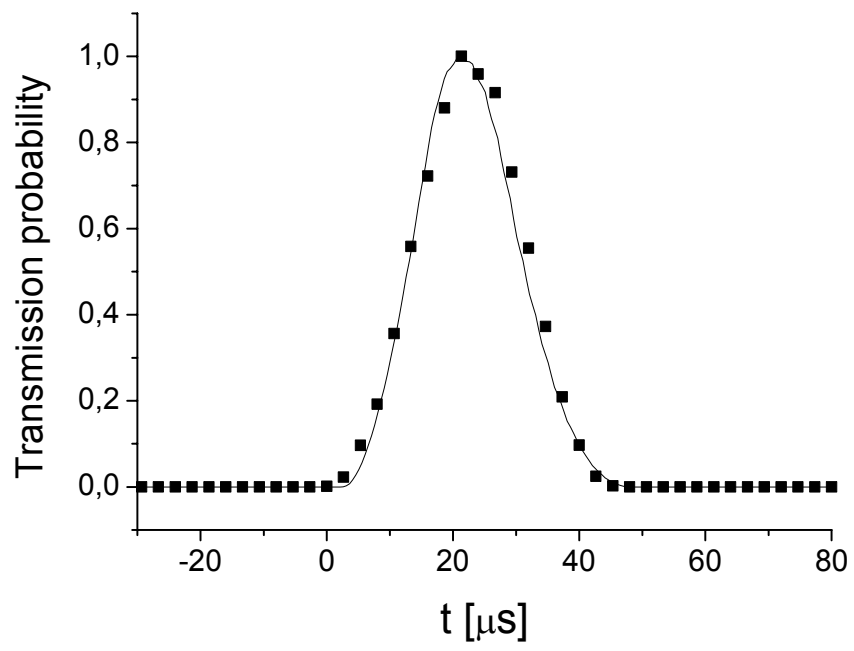


Figure 14: Straight Fermi chopper rotating at 36.000 RPM. Here $0.7 \AA ీ \leq \lambda \leq 1.7 \AA$, the divergence $=$ $1^{\circ}$.

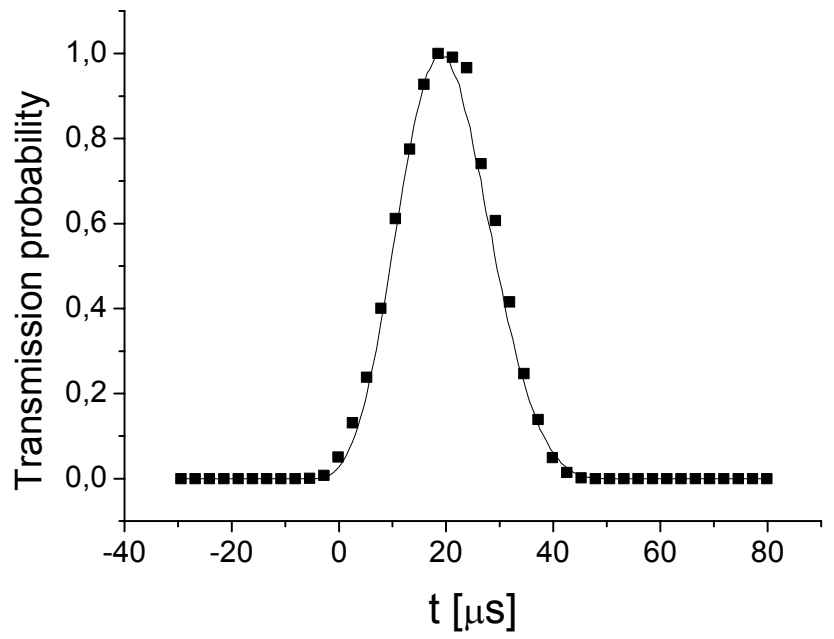

Figure 15: Straight Fermi chopper rotating at 36.000 RPM. Here $0.7 \AA \leq \lambda \leq 1.7 \AA$, the divergence $=$ $3^{\circ}$.

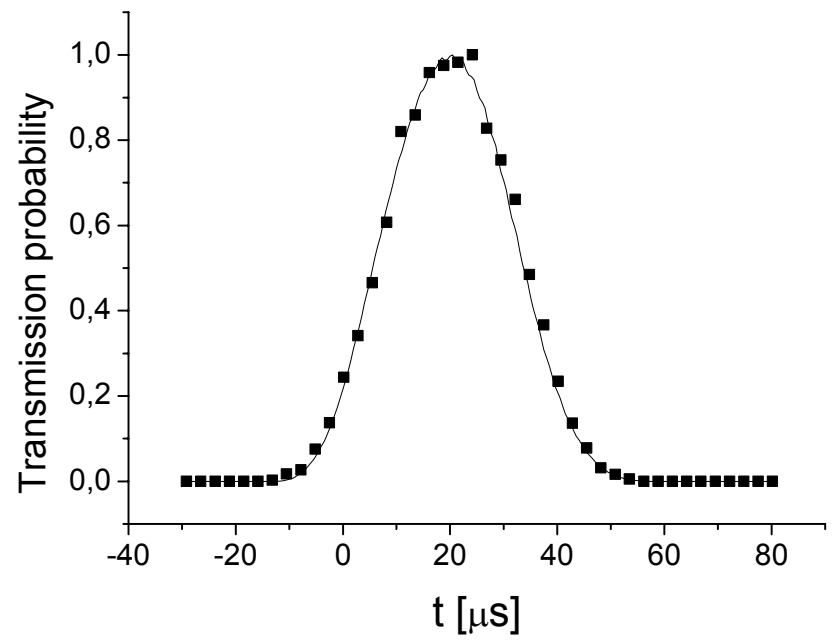


Figure 16: Curved Fermi chopper rotating at 36.000 RPM. Here $\lambda_{0}=1.2 \AA$, the divergence $=0.1^{\circ}$.

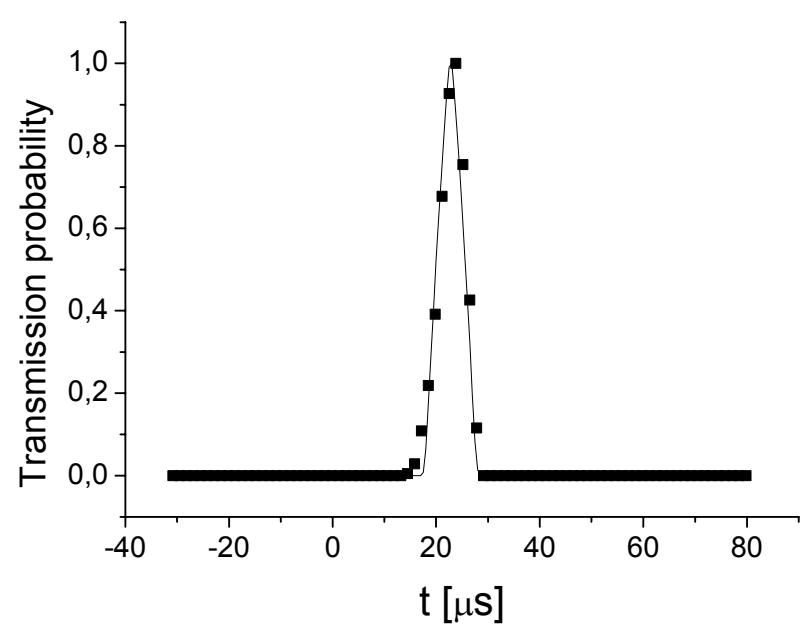

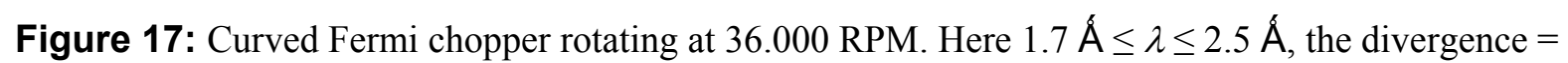
$1^{\circ}$.

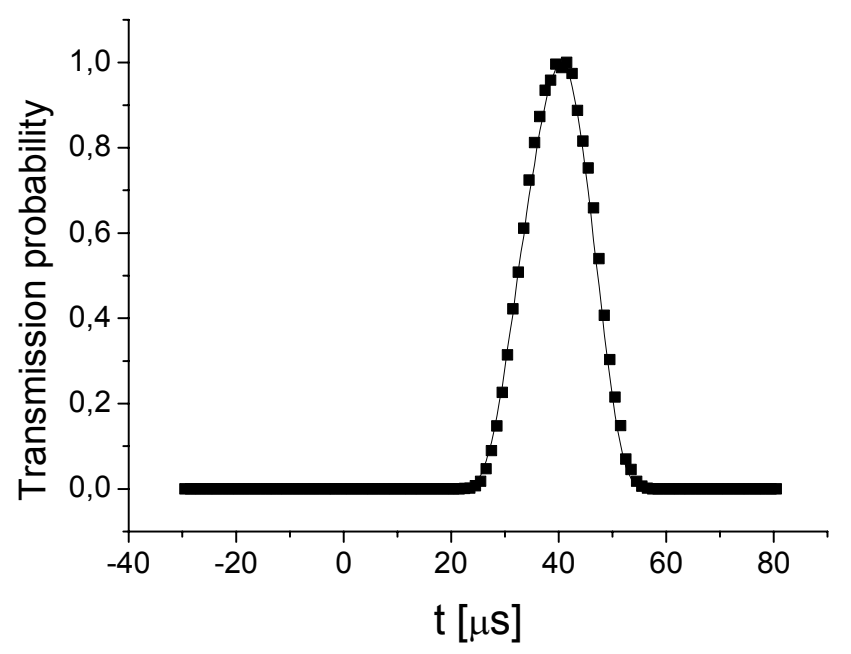


Table 1: Equations (22) for $P(k, t)$ and $\alpha_{e s c}(k, t)$ with the corresponding $k$ and $t$ ranges for a divergent neutron beam.

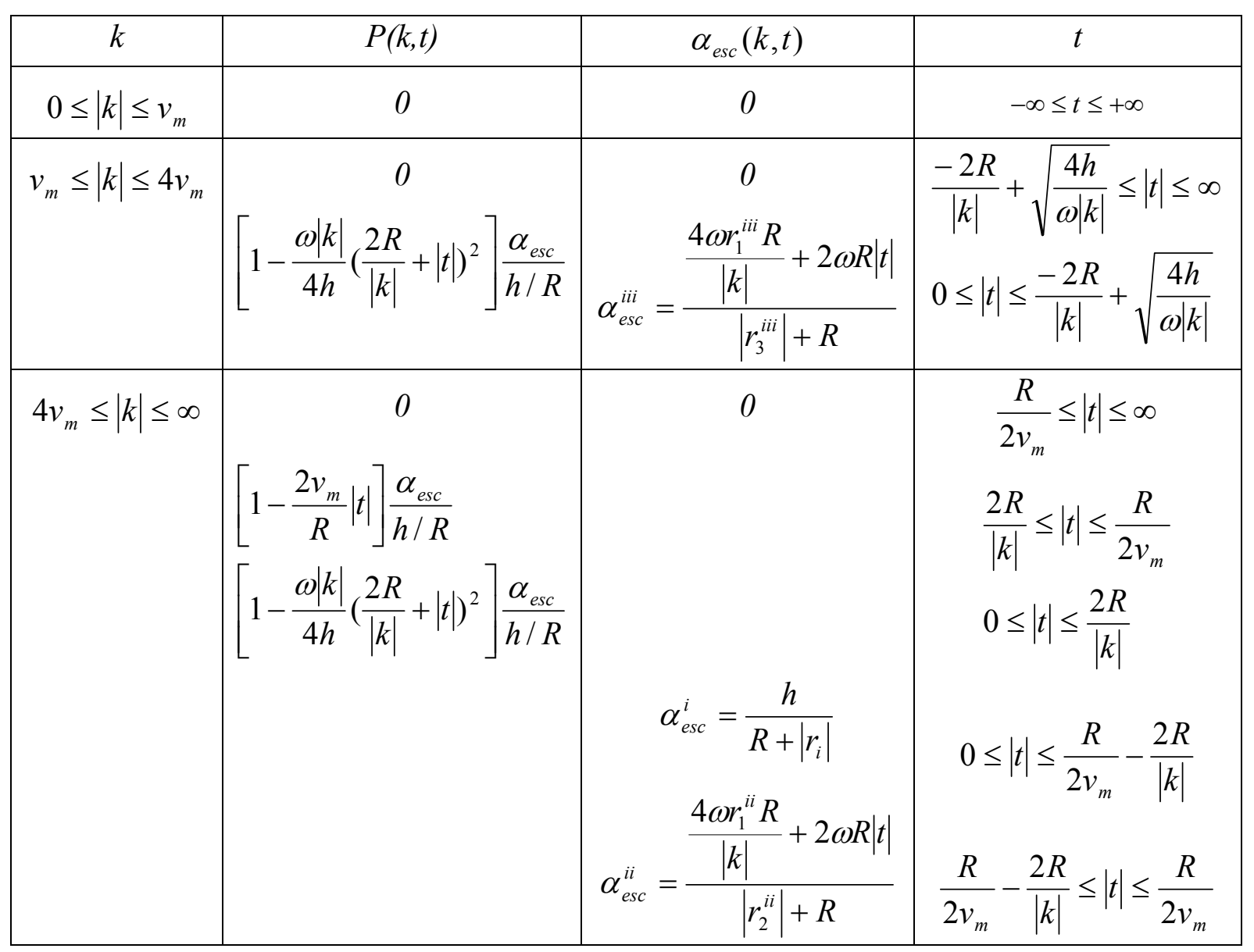

